\title{
Violations of safe diving practices among 122 diver fatalities
}

\author{
Karl Shreeves ${ }^{1}$, Peter Buzzacott ${ }^{2,3}$, Al Hornsby ${ }^{1}$, Mark Caney ${ }^{1}$ \\ ${ }^{1}$ Professional Association of Diving Instructors, Rancho Santa Margarita, California, United States \\ ${ }^{2}$ Medical Research Department, Divers Alert Network, Durham, North Carolina, United States \\ ${ }^{3}$ School of Sport Science, Exercise and Health, the University of Western Australia, Perth, Australia
}

\begin{abstract}
Background: Diving is a popular recreation with an excellent safety record, with an estimated 1.8 deaths per 1 million dives. This study investigated the relationship between intentional deviation from accepted diving practices (violations) and diver fatalities.

Materials and methods: The authors examined 119 incidents/122 diver fatalities that did not involve diver training in North America and the Caribbean, and identified the presence of violations of accepted diving safety practices, as well as if the death was associated with an acute medical event such as heart attack. Results: Of the 122 fatalities, $57 \%(n=70)$ were associated with a medical event and $43 \%(n=52)$ were non-medical. Violations were found in $45 \%$ of fatalities $(n=55)$ overall. Violations were recorded for $23 \%$ of the 70 medical and $75 \%$ of the 52 non-medical fatalities. Divers who died from something other than a medical cause were 7 times as likely to have one or more violations associated with the fatality (OR 7.3, 95\% $\mathrm{Cl}$ 2.3-23.2). The odds of dying from something other than a medical condition increased approximately $60 \%$ for each additional 10 metres of depth. The odds of a death being associated with a medical condition increased approximately $9 \%$ per year of age, or 2.4 times for every 10 years older a diver was. Conclusions: Medical events are associated with over half of the non-training related diver fatalities in North America and the Caribbean, with the odds of death being associated with a medical condition doubling each decade of additional age. These data support recommendations that divers stay physically fit and have regular medical checkups, particularly as they get older. They also strongly support the safety benefit of adhering to established safe diving practices.
\end{abstract}

(Int Marit Health 2018; 69, 2: 94-98)

Key words: health, incidents, Professional Association of Diving Instructors (PADI), risk factors, safety, tourism, unconsciousness

\section{INTRODUCTION}

Although an activity with inherent risks, recreational diving has a low mortality rate, estimated by the Divers Alert Network (DAN) at 1.8 deaths per million dives [1]. In its most recent Annual Diving Report, DAN estimated that for the United States, around 2 out of every 100,000 recreational divers die while scuba diving annually [2]. Cardiac-related medical problems are a leading cause of death in divers and risk factors for an acute coronary event are well documented even in dive professionals, including smoking, overweight/obesity, and high blood pressure [3]. Deviation from established safe diving practices has also previously been found associated with recreational diving mortality $[4,5]$, morbidity and near-miss incidents [6, 7]. Previous researchers in non-diving settings have noted that many unsafe deviations appear to be intentional choices, later thought associated with fatalities [8, 9]. Lagache [10] found $87 \%$ of fatalities in the 1989 Ontario Underwater Council reports had such deviations, with $86 \%$ contributing to the chain of events leading to death.

The intention of this study was to assess what proportion of diver fatality reports (in which it was possible to make a determination) have such intentional deviations. If correlations between intentional deviations and fatalities were 
also found, then it would be another step in understanding behaviours that lead to, or contribute to, diving mortality, and would support further improvements in diver training, supervisory techniques and dive community practices.

\section{MATERIALS AND METHODS}

Two authors reviewed 236 cases involving diving fatalities outside of training in North America and the Caribbean that were reported, as is contractually required, by Professional Association of Diving Instructors (PADI) members during 2010 through August 2016. The reviewers identified cases in which it could be determined, by reported facts or reasonable inference, whether or not a violation of accepted safe diving practices likely occurred and if that violation was causative or merely contributory.

An "accepted safe diving practice" was defined as a behaviour that is taught in diver training courses and accepted in practice by the broad diving community. This study used the definition of "violation" as consciously choosing to not follow a safe diving practice and knowingly creating avoidable risk of harm to self or others. An "error", by comparison, was defined as attempting to follow safe practices, but failing to do so. Both of these definitions are consistent with previous research [11].

In analysing each case, the reviewing authors determined whenever there was a violation by the victim, buddy or other directly involved diver, based on either a violation being reported, or being clearly inferable from the data. If there was no reported violation and/or if all deviations from accepted practices in a particular case could reasonably be explained by circumstances or error (i.e., trying to do the right thing but doing the wrong thing), then no violation was noted. This is similar to the approach previously used $[4,10]$. The reviewers recorded the binary classification of "present" or "absent" in each case, irrespective of the number or type of violations. The reviewers also assessed whether each fatality likely commenced with a medical event in the diver, not directly attributable to diving, such as a heart attack. The determination of each death being associated with a medical event was made by reviewing information in the report, including autopsy, medical information, reported statements by individuals with medical expertise, and/or witness accounts of events that left no other reasonable explanation. Nonetheless, the absence of formal medical reports for all incidents is a study limitation the authors acknowledge.

Fatalities involving divers undertaking training dives were excluded to avoid ambiguity around the choice to follow or deviate from a practice. The majority of cases $(n=113)$ involved recreational no decompression diving, but there were some $(n=9)$ involving technical diving (a specialist sub-set of recreational diving that uses special equipment and procedures to manage risk beyond the limits of mainstream recreational diving).

Case reviews were conducted by the two reviewing authors independently, each going through half the reports. Cases with insufficient detail to provide useful data were excluded ( $n=112 / 236,47 \%$ ), as was one breath-hold case. Data for the remaining cases were tabulated in Excel for analysis. The reviewing authors then reviewed each other's cases, noting any disagreements in classification of violations or medical triggers. Disagreements were noted in $30(13 \%)$ cases. These were resolved for 29 cases, with one discarded due to no clear resolution.

In some instances $(\mathrm{n}=11$ medical events and 10 violations, $9 \%$ of the data), violations and medical events were categorised as "Likely," meaning there was some room for doubt but the reviewing authors considered it more likely than not, or "Possible," meaning considerable room for doubt but the variable could be inferred. In the final analyses, the "Likely" variables were tabulated as present and the "Possible" variables absent. A comparison with and without this categorisation showed no meaningful effect on the results and conclusions.

Lastly, one reviewing author went through the retained fatalities, comparing the reports to the tabulated data to double-check consistency. The end data set included 122 (52\%) fatalities from 119 incidents (there were 3 double fatality incidents), and these are the data described in the results that follow. An application for ethical approval was made to the Institutional Review Board of the Divers Alert Network and this analysis was deemed exempt from needing approval.

\section{RESULTS}

Fatalities were classed as medical $(57 \%, n=70 / 122)$ or non-medical $(43 \%, n=52 / 122)$ events.

Violations were reported or inferable in $45 \%(n=55)$ of the 122 fatalities. Of these 55 fatalities, the violations in $58 \%(n=32 / 55)$ were reported or inferable as being the primary cause, and in $95 \%(n=52 / 55)$ as contributing to the incident. The medical and non-medical groups had significantly different $(p<0.0001)$ proportions of violations. All of the causative medical violations involved diving with a known medical condition that required a physician's clearance before diving, as defined by the Recreational Scuba Training Council (RSTC) medical screen, but not having that clearance. Diving with such a medical condition, but with a physician's clearance, was not considered a violation.

Table 1 summarises the distribution of violations by age and sex, violations group, and incident and maximum depths.

The hypothesised model was that the odds of a fatality in these data being medically related would be associated with 
Table 1. Distribution of violations by age and sex, violations group, and incident and maximum depths

\begin{tabular}{|c|c|c|c|}
\hline & Medical group $(n=70)$ & Non-medical group $(n=52)$ & Overall $(n=122)$ \\
\hline Age $^{a}$ [years] & $61(22-81)$ & $41(15-67)$ & $56(15-81)$ \\
\hline Males $^{b}$ & $53(76 \%)$ & $44(85 \%)$ & $97(80 \%)$ \\
\hline Cases with violations & $16(23 \%)$ & 39 (75\%) & $55(45 \%)$ \\
\hline Violations causative & $4(6 \%)$ & $28(54 \%)$ & $32(26 \%)$ \\
\hline Violations contributive & $13(19 \%)$ & $39(75 \%)$ & $52(43 \%)$ \\
\hline Incident depth ${ }^{c}$ & $0(0-37)$ & $11(0-79)$ & $6(0-79)$ \\
\hline Maximum depth ${ }^{d}$ & $19(0-40)$ & $25(5-79)$ & $20(0-79)$ \\
\hline
\end{tabular}

Data are shown as number (percentage) or median (range).

${ }^{a}$ Age was known in 120 (98\%) fatalities.

bSex was known in all fatalities.

'Depth at which the incident started, in metres of seawater, was known in 95 (78\%) fatalities.

dMaximum dive depth reached, in metres of seawater, was known in 85 (70\%) fatalities.

Table 2. Significance and point estimates of variables in the final model, with 95\% confidence intervals (for the outcome Medical = yes)

\begin{tabular}{lllll}
\hline & Chi-square & Pr $>$ Chi-square & Odds ratio & 95\% confidence intervals \\
\hline Violations (no vs. yes) & 11.48 & 0.0007 & 7.34 & $2.32-23.24$ \\
Incident depth (per msw deeper) & 4.55 & 0.033 & 0.95 & $0.91-1.00$ \\
Age (per year older) & 11.60 & 0.0007 & 1.09 & $1.04-1.15$
\end{tabular}

age, sex, dive depth, incident depth, causative violations and/or any violation. After initial examination, sex, causative violations and dive depth were found least closely associated and were eliminated from the model. The final model is shown in Equation 1, where $\alpha$, and $\beta_{1-3}$ are constants.

$$
\begin{gathered}
\text { Medical (yes or no } \left.)=\alpha+\beta_{1} \text { (Violations }\right) \\
+\beta_{2}(\text { Incident Depth })+\beta_{3}(\text { Age })
\end{gathered}
$$

The $\mathrm{R}^{2}$ was 0.41 , indicating 0.64 correlation, which means the final model accounts for $41 \%$ of the data variability around the mean. The significance of each variable is shown in Table 2.

Based on these data and this model, the authors found that divers who died from something other than an associated medical event (or from an unknown cause) were approximately 7 times as likely to have at least one violation associated with the fatal dive. Or, conversely, divers who died following an associated medical event were one seventh times as likely to have a violation associated with the fatality.

The odds of dying from something other than a known medical event increased 1.05 (5\%) for each metre of depth. In broader increments, for each 10 msw deeper when the incident commenced, the odds of dying from something other than a known medical event increased $64 \%\left(1.05^{10}=\right.$ 1.64). Among the medical cases where depths were known
Table 3. Incident and maximum depths (msw) among non-medical fatalities $(n=52)$

\begin{tabular}{lll}
\hline Range [msw] & Incident depth & Maximum depth \\
\hline $0-9$ & $14(27 \%)$ & $6(12 \%)$ \\
$9-18$ & $6(12 \%)$ & $6(12 \%)$ \\
$18-40$ & $10(19 \%)$ & $13(25 \%)$ \\
$>40$ & $4(8 \%)$ & $7(13 \%)$ \\
Unknown & $18(35 \%)$ & $20(38 \%)$
\end{tabular}

( $n=54)$, only $7 \%$ of these $(n=4)$ had a maximum depth of $0 \mathrm{msw}$, yet $50 \%(n=35 / 70)$ of the incidents involving a medical case initially started on the surface. Maximum dive depths and incident depths in the non-medical cases are shown in Table 3.

The odds of dying in association with a known medical condition increased 9\% for each additional year of age, or approximately doubled every additional 8 years $\left(1.09^{8}\right.$ $=1.99$ ). Moreover, there was a marked difference in median age between medical and non-medical reports, being 61 years vs. 41 , respectively. This study also found $61 \%(n=43)$ of the 70 medical cases had reported or inferable factors consistent with heart disease; these ranged from medical reports and the reported opinions of medical personnel on scene, to signs such as sudden unresponsiveness coupled with age, obesity and/or exertion. 
Tolerated buddy separation or solo diving was reported/inferable in $26 \%(n=32 / 122)$ of cases overall. It was present in $40 \%(n=21)$ of the 52 non-medical cases, and was considered contributive in $38 \%(n=20)$ in that it precluded the possibility of assistance or rescue. The lone exception was a solo technical dive. This was not counted as a violation, because solo diving is accepted (though not universally endorsed) in technical diving. Solo/buddy separation was noted in $16 \%(n=11)$ of the 70 medical cases; of these 9 were considered contributive. The authors noted no incidents in which having a buddy was a causative or contributive factor.

In $41 \%(n=29)$ of 70 medical cases, the victim ended the dive early, (with ample breathing gas remaining and before reaching a planned limit), without an apparent cause or explanation. In these instances the victim commonly became unresponsive during ascent, or shortly after reaching the surface. The authors noted reported signs consistent with Immersion Pulmonary Oedema (IPO), such as frothy expectorant, in $14 \%(n=17)$ of the 122 cases. There was no attempt to further diagnose IPO, because there was no basis for doing so; the authors acknowledge that in some of these cases, the signs may have been explainable due to drowning and other possible causes. The majority of cases with IPO signs $(71 \%, n=12 / 17)$ were fatalities associated with medical events.

Neither age nor sex was found to be closely associated with signs of IPO, likely due to the small data set and/or the inability to further separate IPO from other maladies that cause overlapping signs. The authors noted no meaningful patterns or associations between sex and incident depth, buddy separation/solo diving or violations, with the exception of technical diving and double fatalities. All technical diving exposure cases, (those involving technical diving environments where the victim was not necessarily qualified), and all double fatality victims were male.

\section{DISCUSSION}

The data in this study support the community-held premise that violating safe diving practices is likely one of the primary causes/contributors to diver fatalities, though the degree to which it does remains unquantified. Also, it should be remembered that these data were reported by PADI members in the United States and Caribbean and the results and conclusions may not be applicable to recreational divers in other geographic locations or diving situations.

The authors acknowledge that quantifying violations is problematic and that, doubtlessly, additional violations existed in cases classified either with or without violations. Also, the authors acknowledge that, as stated earlier, quantifying medical events presents a potential study limitation.
Nonetheless, the overall findings are consistent with previous findings $[4,10]$. The maximum depth range was also consistent with DAN dive distribution findings from Project Dive Exploration [12, 13].

The ages of the fatalities in this study are consistent with those reported for 2014, which showed that more than half the deceased were 50 years or older [2]. Given that many individuals continue to dive well into their senior years without incident, it is plausible that it is not age per sé that increases the odds of a medically-related death while diving, but the tendency for risk factors for cardiac disease to increase in prevalence with age $[3,14,15]$. Although comparable, DAN's data are not identical to those analysed in this study because they cover different geographic areas, DAN do not segregate medical/non-medical causes of death and their fatality data include training as well as non-training dives. It is likely that some, but not all, the cases in this study are included in the DAN fatality dataset but the reports compiled by PADI members are not forwarded to DAN and not included in the DAN case files. Therefore, the data reported in this study have not been previously reported.

The data presented in this study appear to support the assertion that diving with a buddy has a risk reduction benefit. Given that $41 \%$ of the medical cases ended the dive early, we suggest that when a diver indicates terminating a dive early then dive buddies stay together all the way to the boat/shore, even in circumstances when it would otherwise be reasonable to observe the diver's ascent and exit from below.

\section{SUMMARY AND RECOMMENDATIONS}

1. Violations of safe diving practices have been found associated with diving incidents of varying seriousness. By comparing medical and non-medical diving fatalities, this study found a demonstrable difference that correlates non-medical fatalities with greater prevalence of at least one safety violation. Our recommendation, therefore, is that divers discipline themselves with respect to following accepted safe diving practices, even when deviating from accepted practices may offer convenience and appear reasonable.

2. Being in good health and fitness for diving is important at any age, but these data highlight the prevailing recommendation that as one gets older, the need to maintain fitness and regular medical assessment becomes increasingly important for risk management.

3. Solo diving (intended or tolerated buddy separation) was not found to be a causative violation, but appeared in $26 \%$ of cases over all, and $44 \%$ of non-medical cases. It was found contributive in the majority of both groups by precluding any possibility of assis- 
tance. No incidents where diving with a buddy was a causative factor were noted. Our recommendation is for recreational divers to adhere to the buddy system, even in circumstances such as technical diving in which solo diving is currently acceptable to many participants. This recommendation is not directed at research, commercial, military or public safety diving, all of which use different protocols.

4. Based on the large proportion of medical case dives that were ended early by the victim (41\%), our recommendation is to maintain the buddy system with a diver who wants to terminate the dive early, even in circumstances where it may be otherwise reasonable to observe the diver's ascent and exit from below.

5. With such a small sample size $(n=122)$ the odds ratios reported in this study may not approximate relative risk among recreational divers. Despite the high proportion of violations among non-medical fatalities, and the causative/contributive role these played, it remains nonetheless likely that not all dives with violations are fatal and that dives with violations are more common than fatal dives. While the data strongly point to a substantial increase in risk due to violations, the increase in absolute risk remains unquantified. Therefore, the authors recommend continuing efforts to gather data on common diving behaviours as well as more detail concerning incidents that get reported. It may be useful to develop an incident reporting system that collects data with the intent of investigating violations/errors and other variables that might not otherwise be apparent, whether they do or do not contribute to the incident. For example, multiple violations were found among Western Australian recreational diving fatalities [4]. A prospective study may determine which violations are the most common, the most serious and/or the more likely to contribute to diving fatalities.

\section{ACKNOWLEDGEMENTS}

The authors wish to thank and acknowledge the following for their explicit support of this research and report: Petar Denoble, MD, DSc, Divers Alert Network, North Carolina, USA; Simon Mitchell, MB, ChB, PhD, DipDHM, DipOccMed, CertDHM (ANZCA), FANZCA, University of Auckland, New Zealand; Drew Richardson, EdD, Professional Association of Diving Instructors, California, USA.

\section{FUNDING}

This work was supported by the Professional Association of Diving Instructors (PADI) and Divers Alert Network (DAN).

\section{REFERENCES}

1. Buzzacott P, Schiller D, Crain J, et al. Epidemiology of morbidity and mortality in US and Canadian recreational scuba diving. Public Health. 2018; 155: 62-68, doi: 10.1016/j.puhe.2017.11.011, indexed in Pubmed: 29306625.

2. Buzzacott P. editor). DAN Annual Diving Report 2016 Edition: A report on 2014 data on diving fatalities, injuries, and incidents. Buzzacott $P$, editor. Durham, NC: Divers Alert Network; 2016. PMID. 28211666.

3. Pougnet R, Costanzo LDi, Loddé B, et al. Cardiovascular risk factors and cardiovascular risk assessment in professional divers. Int Marit Health. 2012; 63(3): 164-169, indexed in Pubmed: 23129099.

4. Buzzacott P, Rosenberg M, Pikora T. Western Australian recreational scuba diving fatalities, 1992 to 2005. Aust N Z J Public Health. 2009; 33(3): 212-214, doi: 10.1111/j.1753-6405.2009.00377.x, indexed in Pubmed: 19630838.

5. Edmonds C, Walker D. Scuba diving fatalities in Australia and New Zealand: The human factor. South Pacific Underwater Medicine Society Journal. 1989; 19: 94-104.

6. Biersner RJ. Factors in 171 navy diving decompression accidents occurring between 1960-1969. Aviat Space Environ Med. 1975; 46(8): 1069-1073, indexed in Pubmed: 1164342.

7. Acott CJ. Human error and violations in 1,000 diving incidents: A review of data from the Diving Incident Monitoring Study (DIMS). South Pacific Underwater Medicine Society Journal. 2005; 35: 11-17.

8. Puchades VM, Pietrantoni L, Fraboni F, et al. Unsafe cycling behaviours and near crashes among Italian cyclists. Int J Inj Contr Saf Promot. 2018; 25(1): 70-77, doi: 10.1080/17457300.2017.134 1931, indexed in Pubmed: 28675090.

9. Freeman J, Rakotonirainy A. Mistakes or deliberate violations? A study into the origins of rule breaking at pedestrian train crossings. Accid Anal Prev. 2015; 77: 45-50, doi: 10.1016/j.aap.2015.01.015, indexed in Pubmed: 25681804.

10. Lagache E. Are divers choosing to die? Undersea Journal. 1993; Fourth Qtr: 50-54.

11. Reason J. Human Error. Cambridge: Cambridge University Press. 1990.

12. Buzzacott P, Denoble PJ, Simon O, et al. Dive problems and risk factors for diving morbidity. Diving Hyperb Med. 2009; 39(4): 205-209, indexed in Pubmed: 22752740.

13. Vann RD, Denoble P, Dovenbarger J, et al. Report on Decompression IIIness, Diving Fatalities and Project Dive Exploration: 2005 Edition (Based on 2003 data). Durham, NC: Divers Alert Network. 2005.

14. Berenji Ardestani S, Buzzacott P, Eftedal I. The aging diver: endothelial biochemistry and its potential implications for cardiovascular health. Diving Hyperb Med. 2015; 45(4): 235-239, indexed in Pubmed: 26687310.

15. Denoble PJ, Marroni A, Vann RD. Annual fatality rates and associated risk factors for recreational scuba diving. In: Vann RD, Lang MA, editors. Recreational Diving Fatalities Worksho Proceedings; Durham, North Carolina: Divers Alert Network. 2010: 73-85. 